\title{
Review Paper: The Resilient Child Indicators in Natural Disasters: A Systematic Review Protocol
}

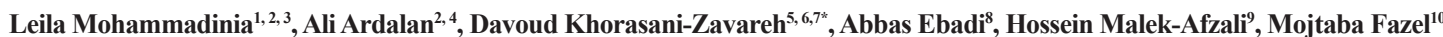

1. Department of Disaster Public Health, School of Public Health, Students 'Scientific Research Center, Tehran University of Medical Sciences, Tehran, Iran.

2. Department of Disaster and Emergency Health, National Institute of Health Research, Tehran University of Medical Sciences, Tehran, Iran.

3. Health Human Resource Research Center, School of Management \&Information Sciences, Shiraz University of Medical Sciences, Shiraz, Iran.

4. Department of Disaster Public Health, School of Public Health, Tehran University of Medical Sciences, Tehran, Iran.

5. Safety Promotion and Injury Prevention Research Center, Shahid Beheshti University of Medical Sciences, Tehran, Iran

6. Department of Health in Disaster and Emergency, School of Health, Safety and Environment, Shahid Beheshti University of Medical Sciences, Tehran, Iran.

7. Department of Clinical Science and Education, Södersjukhuset, Karolinska Institutet, Stockholm, Sweden.

8. Behavioral Sciences Research Center, Faculty of Nursing, Baqiyatallah University of Medical Sciences, Tehran, Iran.

9. Social Determinants of Health Research Center, Tehran Medical Branch, Islamic Azad University, Tehran, Iran

10. Valiasr Hospital, Tehran University of Medical Science, Tehran, Iran.

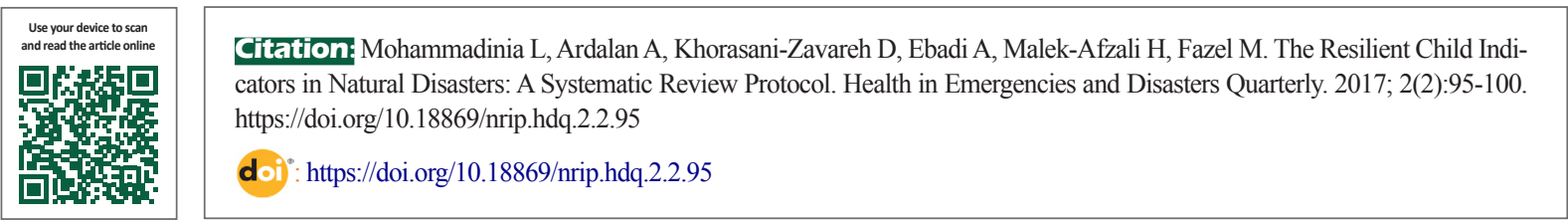

Article info:

Received: 15 Jul. 2016

Accepted: 26 Nov. 2016

\section{Keywords:}

Natural disaster, Resilience, Children, Systematic review

\begin{abstract}
Annually, children as a major group are affected in disasters worldwide. Resilience terminology is appeared in disaster risk reduction to improve more attention paid on human ability instead of concentration on his vulnerability. It seems that child resiliency may be the best approach to decrease the vulnerability. Although there are lots of studies on resiliency, children resiliency in disasters seems to be a unique field to consider. The current systematic review protocol aimed at synthesizing the evidence of the children resiliency indicators in natural disasters to identify their capacity and improve their ability against it. The current systematic review will be conducted on the children resiliency in articles regarding natural disaster situation that extracted indicators/factors to improve children resiliency in natural disasters. Four main electronic databases which cover such articles will be searched including PubMed, Scopus, Web of Science, and Pycinfo. Furthermore, experts will be asked to identify verifiable grey literatures. EndNote software version $\mathrm{X} 7$ will be used to manage searching the databases, screening duplications, and extracting irrelevant articles. Search strategy was defined by the current review authors. Specific syntax was used for each main database. A period will be considered to search the databases. The evaluation of the articles will be conducted based on PICO defined according to the research question to include and exclude the articles. Moreover, the qualitative assessment and review instrument (QARI) checklist from JBI (Joanna Briggs Institute Reviewers' Manual: 2014 edition) will be used to assess the quality criteria. It is a quality context focused on human psychosocial behavior with specific group called children, adolescents, teenagers, and youths, but the current systematic review called them under eighteen-year-old children according to the definition of the United Nations International Children's Emergency Fund (UNICEF). On the other hand, the QARI data extraction instrument will be also used according to the research question.

The current systematic literature review will determine the tangible criteria of child resiliency that would be valuable for decision makers, community, researchers, families, and the people concerned about children and also disaster situations. In this way, in disaster situations they can evaluate the difference between resilient children vs. non-resilient ones; the impacts are limited to the disaster, but also long-time after the disaster.
\end{abstract}

\section{* Corresponding Author: \\ Davoud Khorasani-Zavareh, PhD}

Address: Safety Promotion and Injury Prevention Research Center, Shahid Beheshti University of Medical Sciences, Tehran, Iran.

E-mail: davoud.khorasani@gmail.com 


\section{Introduction}

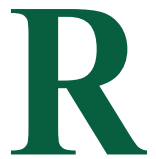

isk, disaster, emergency and risk of natural, health, environmental, and terrorist hazards are increasing in the world [1]. The children of the world are regularly exposed to environmental hazards, both natural and man-made disasters. In the previous 2 decades more than 100 million children were affected by hazards. [2] According to united nations international children's emergency fund (UNICEF) (2013), the number of under 18 years affected children increased up to 2.5 billion by 2050 [3]. On the other hand, the increasing trend of risks and hazards in recent years led the policy makers and researchers to resilience and Disaster Risk Reduction (DRR) concepts to reduce disaster risks that this new approach is on the positive side of events.

In the recent decade, $\mathrm{UN}$ frameworks such as Hyogo and Sendai that concentrated on DRR call the core value in resilient literature focused on children in disaster-resilient communities [4-6]. There are lots of studies on resiliency [7-14] that explain the concepts such as community, society, and individual resiliency, but studies on resilient indicators for specific groups such as children are very rare. For instance, resilience is defined as a process of successful adaptation ability in threatening situations; in other words, a positive adjustment is in response to adverse conditions. Resilience is achieved by tenacity, self-enhancement, coercive coping, positive moods, and feelings [15]. According to Tango (2012), resilience is "individual, community and institution ability to anticipate, withstand, and bounce back from external pressures and shocks in ways that avoid deepening vulnerability" [4]. Children are important groups that should be considered in disasters due to their vulnerability and special potential ability to contribute in disaster risk reduction programs. It is noteworthy that children can create safety and prevention culture in society [16].

Although resiliency has 2 sides, vulnerability and capacity, it seems that most of the scientists focus on vulnerability instead of capacity, similar to most studies [1722]. As capacity issue is rarely studied, the current study aimed at investigating it. The current meanings of resilience are general issues that are not clear to be managed and find out main factors to improve children resiliency. Therefore, the meanings should undergo a concept analysis to define clear indicators understandable to decision makers in disasters.

As there was no systematic review of child resiliency in natural disasters, the current study aimed at examining the studies on this topic. Therefore, the main objective was extracting tangible criteria to show up children resiliency in natural disasters. Generally, the current study findings clarify the future studies on children in disaster situation, which are worth to follow-up both by researchers and policy makers. Due to the importance of child resiliency as a vulnerable group, not highlighted in literatures, the current study aimed to do it.

\section{Materials and Methods}

\section{Study design}

The current study conducted a systematic review protocol on children resiliency in natural disaster situation in any methodological articles that extracted indicators/ factors to improve children resiliency in natural disasters. Four main electronic databases which cover the articles on this topic will be searched: PubMed, Scopus, Web of Science, and PsycInfo. Furthermore, experts will be asked to identify verifiable grey literatures. EndNote software version X7 are going to be used to manage search library, screen duplications, and extract irrelevant articles. Search strategy will be defined by the authors. Specific syntax will be used for each main database. Time limits would be considered for database searching. Articles will be evaluated based on PICO defined according to the research question to include and exclude the articles. Moreover, the qualitative assessment and review instrument (QARI) checklist/tool from JBI (Joanna Briggs Institute Reviewers' Manual: 2014 edition) will be used to assess criteria quality. It will be a quality context and focused on human psychosocial behaviors on a specific group called children, adolescence, teenager, and youths; but the current study will call them under 18-year-old children, according UNICEF definition. On the other hand, the current study will use the QARI data extraction tool according to the research question. Systematic literature reviews in English and without any restrictions of articles 'methodology will be included in the review. The designee will be followed base on the PRISMA statement diagram [23]. PRISMA-P checklist will be employed to assess the eligibility of the included articles. Nowadays, due to the lack of protocol guidance, researchers use PRISMA-P [24]. This checklist evaluates three main sections of articles such as administrative information, introduction, and methods [24].

\section{Study registration}

The current systematic review was registered with PROSPERO (registration number: CRD42016047232; http://www.crd.york.ac.uk/PROSPERO/) 


\section{Inclusion and exclusion criteria}

To answer the review question- the child resilience dilemmas and indicators in natural disasters- the studies on 3 main keywords (resiliency, children and natural disaster) will be selected. All articles on children resiliency will be considered disregarding the methodology, date, and language limitations. It means that studies in English with all types of methodologies will be included in the current systematic review that most of them have good quality. Researchers intende to respond to the review question on child resilience with respect to psychosocial, physical, and environmental approach in natural disaster realm. Furthermore, all research conducted in context of natural disaster will be included and all manmade disasters and any kind of emergencies or clinical studies will be excluded, too.

\section{Population criteria}

The current review specifically focuses on 6- to 18-year-old children.

\section{Type of interventions}

Any program or indicator that described resiliency in children in natural disaster situation as an eligible intervention will be included in the current review. Furthermore, all suggestion to improve child resiliency will be considered, too. Resiliency has diversity domain; therefore, mental health and psychological aspects are important and social, cultural, and environmental indicators are included. The study will restrict the inclusion documents only to "natural disaster". The documents should describe children resiliency as a theoretical or scientific description to define eligible criteria to improve children resiliency in disaster situations.

\section{Data collection}

\section{Search methods to identify studies or search strategy}

The search process in the current review will consist of 3 steps by JBI's CReMS [25] standard. First of all, computerized databases will be searched without any date and language restriction. The keywords will be selected according to Mesh entry, expert opinion, and similar study keywords. Scopus, Web of Science, Medline, and PsycInfo will be used as databases. In addition, search strategy for each database will be defined by expert panel team. The search terms and strategies will be as follows: [(Resilient* and (Child* or youth or adolescent* or young or teen*) and disaster, title, abstract, head words, table of contents, key concepts, original title, tests, and measures].
Then, special databases and grey literatures will also be searched through global platform for DDR, ERIC, fosteringresilience.com, and 6th international building resilience conference. Moreover, covering adhesion searched these websites - unicef/prevention web/unisdr/mhpss will be followed to extract data according to foreign expert suggestions by face-to-face interview at international conferences, emails, and also Skype interviews. Finally, the reference list of all identified reports and articles will be checked to evaluate any missing additional studies.

\section{Results}

\section{Selection of studies}

All search results will be transferred into EndNote software. First of all, duplication documents will be deleted. Then, data evaluation will start by title, abstract, and keywords by inclusion criteria. Full texts of the remained articles will be searched. They will be screened with the inclusion and exclusion criteria and also standard quality assessment [23-25] will be conducted. Two authors will assess eligibility, independently. In case of any disagreements between the 2 reviewers, the third author will resolve the case.

\section{Data extraction}

The search terms for each database will be defined according to the research questions and several peer-review discussions. The syntax of keywords will be employed based on the number of non-replicated (NNR) index. LM will carry out the initial search of studies using the databases listed above in cooperation with DKZ as the supervisor. Titles and abstracts will be independently screened for inclusion in the systematic review and discrepancies by 2 researchers. LM will screen and code the full publications and will exclude any articles that would not meet the inclusion criteria and discrepancies will be resolved by discussion.

\section{Risk of bias assessment}

Quality assessment of the selected studies will be carried out by the QARI checklist based on JBI [25] recommended by Cochrane group. Quality assessment will be conducted by 2 authors and studies will be selected accordingly.

\section{Discussion}

\section{Data synthesis and analysis}

Contextual analysis will be used for the collected data. It has predicted to do subgroup or subset analysis in 2 
resilient domains such as physical, psychosocial, etc.; although it is dynamic through the processes. Finally, holistic definition of children resiliency in disaster context will be described in psychology context in addition to the other context.

\section{Conclusion}

A new prospect of children resilience in disaster will be provided in the main systematic review and its terminology in disaster context will be defined with multiple dimensions and also the tangible indicators of children resiliency which will be extracted for disaster managers and policy makers to consider before and after disaster planning. Therefore, it has primary and secondary outcomes such as: paying attention to children in disaster situations and defining the resilience dilemma for children in disaster situations. The final result will clarify the diagnostic tangible indicators of resiliency of children spatial for disaster situation and clear factors for policy makers and disaster managers about children.

\section{Limitation}

The main limitation was authors ' lack of access to the main famous and essential databases due to the economic sanctions against the country. It took a long time to receive open access through the 1st author's close friend who lived abroad.

\section{Acknowledgements}

This article is part of a study which is conducting in Tehran University of Medical Sciences, Iran. The researchers hereby thank the School of Public Heath, Department of Health in Disasters and Emergencies which are supporting the study.

\section{Conflict of Interest}

The authors declared no conflicts of interests.

\section{References}

[1] Cutter SL, Ahearn JA, Amadei B, Crawford P, Eide EA, Galloway GE, et al. Disaster resilience: A national imperative. Environment: Science and Policy for Sustainable Development. 2013;55(2):25-9. doi: 10.1080/00139157.2013.768076

[2] Kamath SS. Child protection during disasters. Indian pediatrics. 2015; 52(6):467-8. doi: 10.1007/s13312-015-0656-4
[3] UNICEF. Child rights toolkit; Child rights in crisis and riskprone situations. Indonesia: European commission. Indonesia: UNICEF; 2013.

[4] UNICEF. UNICEF and child-centred disaster risk reduction 2009 [Cited 2016 July 20]. Available from: http:/ / www. unicef.uz/upload/iblock/95c/2_child_centred-drr.pdf

[5] The International Federation of Red Cross and Red Crescent Societies (IFRC). World disasters report 2015: Focus on local actors, the key to humanitarian effectiveness. Report No. 97892-9139-226-1. Geneva: The International Federation of Red Cross and Red Crescent Societies; 2015

[6] United Nations Office for Disaster Risk Reduction (UNISDR). Sendai framework for disaster risk reduction 2015-2030. Report No.: 15-05383. New York: United Nations; 2015.

[7] Shimizu M. Resilience in Disaster Management and Public Policy: A Case Study of the Tohoku Disaster. Risk, Hazards \& Crisis in Public Policy. 2012; 3(4):40-59. doi: 10.1002/rhc3.17

[8] Masten AS. Global perspectives on resilience in children and youth. Child Development. 2013; 85(1):6-20. doi: 10.1111/ cdev.12205

[9] Masten AS, Narayan AJ. Child development in the context of disaster, war, and terrorism: Pathways of risk and resilience. Annual Review of Psychology. 2012; 63(1):227-57. doi: 10.1146/annurev-psych-120710-100356

[10] Ostadtaghizadeh A, Ardalan A, Paton D, Jabbari H, Khankeh HR. Community disaster resilience: A systematic review on assessment models and tools. PLoS currents. 2015; 7. doi: 10.1371/currents.dis.f224ef8efbdfcf1d508dd0de4d$8210 \mathrm{ed}$

[11] Paton D, Johnston DM. Disaster resilience: An integrated approach. Springfield: Charles C Thomas Publisher; 2006.

[12] Paton D. Disaster resilience: integrating individual, community, institutional and environmental perspectives. In: D. Paton, D. Johnston editors. Disaster Resilience: An integrated approach. Springfield: Charles C Thomas Publisher.

[13] Ostad Taghizadeh A, Ardalan A, Paton D, Jabbari H. Community disaster resilience: A qualitative study on Iranian concepts and indicators. Natural Hazards. 2016; 83(3):1-19. doi: 10.1007/s11069-016-2377-y

[14] Paton D, Selway K. L., Mamula-Seadon L. Community Resilience in Christchurch: Adaptive responses and capacities during earthquake recovery. Avalon: Institute of Geological and Nuclear Sciences; 2013.

[15] Atadokht A, Norozi H, Ghaffari O. [The effect of socia problem-solving training on psychological well-being and resiliency of students with learning difficulties (Persian)]. Journal of Learning Disabilities. 2014;3(2)

[16] Masten AS. Pathways to integrated resilience science. Psychological Inquiry. 2015; 26(2):187-96. doi: 10.1080/1047840x.2015.1012041

[17] Berg BM, Musigdilok VV, Haro TM, Myers P. Public-Private Partnerships: A whole community approach to addressing children's needs in disasters. Clinical Paediatric Emergency Medicine. 2014;15(4):281-8. doi: 10.1016/j.cpem.2014.10.003

[18] Tol WA, Song S, Jordans MJ. Annual research review: Resilience and mental health in children and adolescents living 
in areas of armed conflict-a systematic review of findings in low-and middle-income countries. Journal of Child Psychology and Psychiatry. 2013; 54(4):445-60. doi: 10.1111/jcpp.12053

[19] Hersi HAF. NGO roles in disaster preparedness and mitigation (DPM): A case study of Save the Children UK in drought-prone Fik, Ethiopia [MSc. thesis]. Ann Arbor: University of Guelph; 2003.

[20] Eksi A, Braun KL. Over-time changes in PTSD and depression among children surviving the 1999 Istanbul earthquake. European Child \& Adolescent Psychiatry. 2009; 18(6):384-91. doi: 10.1007/s00787-009-0745-9

[21] Pfefferbaum B, Maida CA, Steinberg AM, Beaton RD, Pynoos RS, Fairbank JA, et al. Enhancing national capacity to conduct child and family disaster mental health research. Nursing Education Perspectives. 2010; 31(4):237-41. PMID: 20882865

[22] Bronstein I, Montgomery P. Psychological distress in refugee children: A systematic review. Clinical Child and Family Psychology Review. 2011; 14(1):44-56. doi: 10.1007/s10567010-0081-0

[23] Moher D, Liberati A, Tetzlaff J, Altman DG, Group P. Preferred reporting items for systematic reviews and meta-analyses: the PRISMA statement. International Journal of Surgery. 2010; 8(5):336-41. doi: 10.1016/j.ijsu.2010.02.007

[24] Shamseer L, Moher D, Clarke M, Ghersi D, Liberati A, Petticrew M, et al. Preferred reporting items for systematic review and meta-analysis protocols (PRISMA-P) 2015: Elaboration and explanation. BMJ. 2015; 349:g7647. doi: 10.1136/bmj. g7647

[25] Institute JB. Joanna Briggs Institute reviewers' manual 2014 edition. Adelaide: Adelaide University; 2014. 
